\title{
Direct Production of Human Cardiac Tissues by Pluripotent Stem Cell Encapsulation in Gelatin Methacryloyl
}

Petra Kerscher ${ }^{1}$, Jennifer A. Kaczmarek ${ }^{1, \#}$, Sara E. Head ${ }^{1, \#}$, Morgan Brazel ${ }^{1, \#}$, Wen J. Seeto ${ }^{1}$, Joonyul Kim², Subhrajit Bhattacharya ${ }^{3}$,Vishnu Suppiramaniam ${ }^{3}$, Elizabeth A. Lipke ${ }^{1, *}$

\section{Affiliations:}

${ }^{1}$ Department of Chemical Engineering, Auburn University, Auburn, AL, USA.

${ }^{2}$ Department of Chemistry and Biochemistry, Auburn University.

${ }^{3}$ Drug Discovery and Development, Harrison School of Pharmacy, Auburn University.

${ }^{\#}$ Equal contributors.

*Corresponding author, Email: elipke@,auburn.edu

\section{Supplementary Figures}

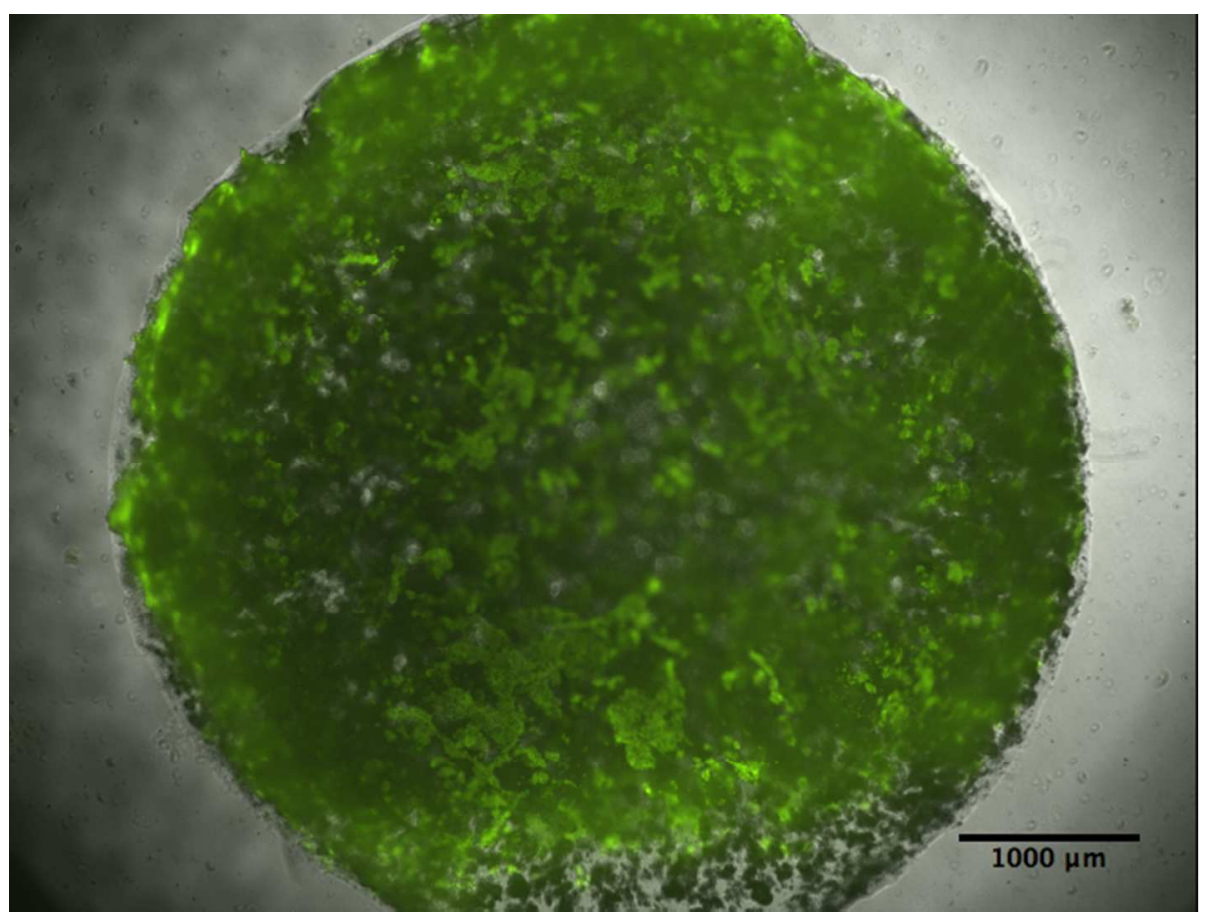

Supplemental Figure 1: Encapsulated cells remain viable following cardiac differentiation.

Overlay of live (green) and phase contrast images from day 8 of differentiation. 